\title{
Cytokine Dependent Hematopoietic Cell Linker (CLNK) is Highly Elevated in Blood Transfusion Dependent Beta-Thalassemia Major Patients
}

Hussein Kadhem Al-Hakeim (1); Hawraa Hussein Al-Mayali (2); Michael Maes (3, 4, 5)

(1) Professor in Medical Chemistry, Department of Chemistry, College of Science, Kufa University-Iraq. headm2010@yahoo.com.

(2) MSc in Biochemstry, Department of Chemistry, College of Science, Kufa University-Iraq. hawaraalmyaly1@gmail.com.

(3) Department of Psychiatry, Faculty of Medicine, Chulalongkorn University, Bangkok, Thailand.

(4) Department of Psychiatry, Medical University Plovdiv, Plovdiv, Bulgaria.

(5) IMPACT Research Center, Deakin University, Geelong, Australia.

Corresponding author

Prof. Dr. Maes, M.D., Ph.D.

IMPACT Strategic Research Center, Barwon Health

Deakin University

Geelong, Vic

Australia.

E-mail: dr.michaelmaes@ hotmail.com 


\begin{abstract}
Beta-thalassemia major $(\beta-\mathrm{TM})$ is a severe form of thalassemia caused by mutations in the $\beta$ globin gene, resulting in partial or complete deficiency of $\beta$-globin chains. This deficiency results in oxidative stress, dyserythropoiesis, and chronic anemia. Cytokine dependent hematopoietic cell linker (CLNK) belongs to the adaptor protein family and has the capacity to interact with multiple signaling proteins thereby modulating signal transduction.

The aim of the present study was to examine CLNK in sera of $\beta$-TM patients and examine its association with iron overload biomarkers.

Sixty $\beta$-TM patients, aged 3-12 years old and undergoing blood transfusions, and 30 healthy control children were recruited and CLNK, ferritin and iron status parameters were measured.

The results showed a significant increase $(\mathrm{p}<0.001)$ in serum CLNK levels in $\beta$-TM patients as compared with normal controls. The increased levels of CLNK were significantly associated with increased ferritin levels.

Increased CLNK levels in $\beta$-TM may be explained by reciprocal effects between immune signaling and immature erythrocytes, which, release soluble receptors and signaling molecules, including CLNK, in the blood.
\end{abstract}


Keywords: CLNK, thalassemia, ferritin, iron overload. 
Introduction

Beta-thalassemia major ( $\beta$-TM), a hereditary anemia characterized by absent or decreased production of $\beta$-globin chains, is associated with considerable morbidity and mortality. ${ }^{1}$ These patients are treated with continuous administration of blood transfusion to raise the depletion of red blood cells $(\mathrm{RBCs}) .^{2}$ The treatment regimen causes iron overload whereby excess iron accumulates in the body organs tissues such as heart, liver and endocrine glands causing damage to the organs. ${ }^{3,4}$ Eryptosis (suicidal erythrocyte death) contributes to or even accounts for the anemia in $\beta$-TM, and involves $\mathrm{Ca}^{2+}$ entry and ceramide, caspase, and tyrosine kinase signaling. Inhibitors of eryptosis may prevent anemia in clinical conditions associated with enhanced eryptosis. ${ }^{5}$ Fetal hemoglobin $(\mathrm{HbF})$, which consists of two $\gamma$-globin chains and two $\alpha$-chains, is normally replaced by adult hemoglobin $(\mathrm{HbA})$ at birth, whilst in $\beta$-TM, $\gamma$-chain production continues into adulthood. ${ }^{6}$ In patients with $\beta$-TM, cAMP levels are elevated in both red blood cells and nucleated erythroblasts and the transcription factor cAMP response element binding protein is phosphorylated in nucleated erythroblasts, while its phosphorylation levels correlate with HBG mRNA levels of the patients. ${ }^{7}$ Many signaling molecules, such as mitogen-activated protein kinases and signal transducers and activators of transcription proteins, are phosphorylated at variable levels. ${ }^{7,8}$ Plasma levels of cytokines, such as erythropoietin, stem cell factor and transforming growth factor- $\beta$ are increased in $\beta$-TM patients, and these cytokines induce both $\gamma$ globin gene (HBG) mRNA expression and cAMP response element binding protein phosphorylation. ${ }^{7}$

The Src family of protein tyrosine kinases (SFKs) is a family of non-receptor tyrosine kinases which play a central role in the regulation of hematopoietic cell functions and is are involved in regulation of membrane transport. ${ }^{9}$ SFKs play a key role in regulating signal 
transduction by a diverse set of cell surface receptors. SFKs exhibit many molecular strategies to couple receptors with the cytoplasmic signaling machinery. ${ }^{10}$ Src-family kinases are implicated in regulation of erythrocyte membrane cation transport including tyrosine phosphorylation regulating $\mathrm{K} / \mathrm{Cl}$ cotransport. ${ }^{9}$

Cao et al., (1999) ${ }^{11}$ have identified a Src homology 2 domain-containing leukocyte protein of $76 \mathrm{kD}$ (SLP-76)-related molecule which they have termed CLNK (cytokine-dependent hemopoietic cell linker). Hematopoietic precursors secrete numerous regulatory molecules that form the basis of the intercellular cross-talk networks regulating in an autocrine and/or a paracrine manner various stages of normal human hematopoiesis. ${ }^{12}$ CLNK, the third member of the SLP-76 family, is a cytokine inducible (IL-2 and IL-3) adaptor protein. ${ }^{13}$ CLNK is expressed exclusively in cytokine-stimulated hemopoietic cells, including IL-2-induced T cells and NK cells, and IL-3-induced myeloid cells and mast cells. ${ }^{14}$ CLNK may undergo tyrosine phosphorylation in response to immunoreceptor, but not cytokine, stimulation. ${ }^{11}$ Inhibition of tyrosine dephosphorylation may alter the shape of RBCs into echinocytes, indicating loss of interaction between the cytoskeleton and lipid bilayers, and stimulates microvesicle production in vitro. ${ }^{15,16,17}$ Nevertheless, no studies have examined CLNK in $\beta$-TM patients.

Hence, the present study examines serum levels of CLNK in $\beta$-TM patients as compared with healthy controls and the associations between CLNK levels and serum iron status. The specific hypothesis is that serum CLNK is significantly increased in $\beta$-TM children.

Subjects and Methods

Subjects 
Sixty $\beta$-TM patients aged 3-12 years participated in the present study. All of them were on blood transfusion regimen in the Thalassemia Unit at "Al-Zahra'a Teaching Hospital" in Najaf city, Iraq. The diagnosis was made by hematologists according to ICD-10-CM Diagnosis Code D56.1 (2019). Moreover all patients showed haematological tests and haemoglobin HPLC analysis indicative of $\beta$-TM. Haemoglobin HPLC were done by using (VARIANTTM $\beta$-T Short Program) HPLC instrument. Normal range for HbA2 is typically between 1.75 and $3.25 \%$ of total haemoglobin while heterozygous $\beta$-T conditions yield $\mathrm{HbA} 2$ levels between 4.0 and 9.0\%. The normal range for $\mathrm{HbF}$ is typically less than $1 \%$ of the total haemoglobin. Heterozygous and homozygous condition of $\beta-\mathrm{T}$ yield $\mathrm{HbF}$ ranges of $1-5 \%$ and $80-100 \%$, respectively. ${ }^{18}$

All patients were treated with blood transfusions and received approximately $15 \mathrm{ml}$ of packed red blood cells/kg of body weight at each transfusion (2-6 week intervals) to maintain $\mathrm{Hb}$ levels above $9.5 \mathrm{~g} / \mathrm{dl}$. Patients were treated with chelation therapy with desferrioxamine B (Desferal) at least four times a week (subcutaneous infusion). The range of dose was 30 to 60 $\mathrm{mg} / \mathrm{kg}$ body weight/day. The median duration of $\beta$-TM was 6.8 years with a range of 2.1 to 9.1 years. The median of the duration of treatment was 4.2 with a range from 1.2-8.3 years. Serological serum CRP was negative in all samples i.e. less than 6mg/L. CRP test was used to exclude inflammation that causes changes in the acute phase reactant proteins. ${ }^{19}$ None of the patients had undergone splenectomy. Endocrinologic, hepatologic and cardiac evaluations were regularly performed and showed normal results at the time of sample collection. Blood samples from patients were collected 7-10 days after the last transfusion and just before the next transfusion. Only patients who are free from any other systemic disease were participated in the study. Thirty apparently healthy children were carefully chosen as the control group. Their age range and mean were comparable to that of patients. None of the controls was anaemic or had 
any obvious systemic or hematological diseases. The study was approved by the local IRB (institutional review board) of the University of Kufa (REC number: 1321). All participants included in this study and their first degree relatives (or guardians) gave written informed consent after appropriate explanation according to the Declaration of Helsinki.

\section{Methods}

Five milliliters of blood was collected from all selected children and separated into two aliquots. One aliquot was transferred into an EDTA tube for hematological analysis and the rest was transferred into a plain tube in order to separate serum by centrifugation at 3000rpm for 10 minutes. The Human CLNK quantitative test (Bioassay Technology Laboratory ${ }^{\circledR}$, China) is based on a solid-phase enzyme-linked immunosorbent assay (ELISA). Serum ferritin concentration was determined by using Vidas Ferritin; an automated quantitative test using enzyme linked fluorescent assay (ELFA) technique. The fluorescence intensity was measured by automated analyzer (miniVidas, Biomeraux-France). Serum iron and TIBC were estimated by kits supplied by Biolabo, France. The other iron status-related parameters were computed using formulas as explained previously: ${ }^{19,20}$ unsaturated iron-binding capacity (UIBC), the amount of protein (apotransferrin) still available to bind iron, can be estimated from the formula: UIBC $=$ TIBC - Serum iron. Transferrin saturation percentage (TS\%) was calculated as: $\mathrm{TS} \%=($ Serum Iron/TIBC $) * 100 \%$. Transferrin concentration can be calculated using the following formula: Transferrin Conc. $(\mathrm{g} / \mathrm{L})=\mathrm{S}$.Iron $(\mu \mathrm{mol} / \mathrm{L}) /(\mathrm{TS} \% * 3.98)$. Estimated total iron body stores (ETIBS) were calculated using the following equation: ETIBS (in $\mu \mathrm{mol})=($ Serum ferritin in $\mu \mathrm{g} / \mathrm{L}) \times 143$. 
Statistical Analysis

Chi square test $\left(\chi^{2}\right.$ test) was used to check associations between categorical variables and analysis of variance (ANOVAs) to check differences in continuous variables between groups. Ln transformations were used to normalize the distribution of variables were needed. Multivariate general linear model (GLM) analysis was used to examine the effects of diagnosis ( $\beta$-TM versus controls) on the different biomarkers, which were entered as dependent variables. Power analysis, using a 2-tailed test at $\alpha=0.05$ and assuming an effect size of 0.3 with power of 0.80 , shows that the required sample size is 90 participants. We computed two $\mathrm{z}$ unit weighted composite scores containing $\operatorname{Ln}(\mathrm{CLNK})$ namely, $\mathrm{zLn}(\mathrm{CLNK})+\mathrm{zLn}($ Ferritin) and $z \operatorname{Ln}(\mathrm{CLNK})+z \operatorname{Ln}($ Ferritin) $+\mathrm{zLn}($ Iron)). Receiver operating characteristic (ROC) curves were utilized to determine the sensitivity and specificity of the outcome variables to differentiate between patients and controls. The bootstrapped $(n-=2000)$ Area Under the Curve (AUC) was calculated to determine the diagnostic accuracy for $\beta$-TM. An optimal diagnostic tool has an AUC of 1 , which indicates $100 \%$ sensitivity and specificity. ${ }^{21}$ Tests were 2-tailed and a p-value of 0.05 was used for statistical significance. All statistical analyses were performed using IBM SPSS windows version 25, 2017.

Results

Table 1 presents the socio-demographic, clinical data, iron status parameters, as well as CLNK and the composite scores in $\beta$-TM patients and controls. There were no significant differences in the sex ratio between the both categories, while age was somewhat higher in controls than in patients (without p-correction). $\mathrm{Hb}$ and PCV were significantly lower in $\beta$-TM as compared with controls. Serum iron, TS\%, ferritin, and EIBC were significantly higher in $\beta$-TM 
than in controls. CLNK and the CLNK-containing composite scores were significantly higher in patients as compatred with healthy controls. In the total study group, we found significant correlations between CLNK and $\mathrm{Hb}(\mathrm{p}=-0.341, \mathrm{p}=0.001)$, PCV\% $(\mathrm{p}=-0.338, \mathrm{p}=0.001)$, ferritin $(\mathrm{p}=0.452, \mathrm{p}<0.001)$, but not iron or transferrin. In $\beta$-TM there was a significant association between CLNK and number of transfusions ( $\mathrm{p}=0.305, \mathrm{p}=0.018)$.

Table 2 displays the results of a multivariate GLM analysis with CLNK and the zCLNK+zFerritin+zIron composite score as dependent variables and with diagnosis as primary explanatory variable, while we adjusted for possible age and sex effects. There was a highly significant effect of diagnosis with an effect size of 0.635 , while age and sex had no significant effect on the biomarkers. Tests for betwee-subject analyses showed that there are strong associations between diagnosis and both CLNK and the composite score. We also performed an univariate analysis with CLNK as dependent variable and found that the significant effects of diagnosis were no longer significant after inducing ferritin in the analysis. The last univariate analysis in Table shows that $20.4 \%$ of the variance in CLNK was explained by ferritin.

The results of ROC analysis including AUC are presented in Table 3. The bootstrapped area under the ROC curve was 0.738 for CLNK while that of ferritin was 0.978. Combining CLNK with ferritin and / or iron did not improve the diagnostic performance of ferritin.

\section{Discussion}

The major finding of this study is that CLNK and the CLNK-containing composite scores showed a highly significant increase in patients as compatred with healthy controls. Moreover, the increased levels of CLNK were explained at least in part by increased levels of ferritin. The increased CLNK levels in $\beta$-TM patients may be explained by reciprocal effects of aberrant 
immune signaling coupled with the immaturity of RBCs. In $\beta$-TM, many RBCs in the blood stream contain nucleic acids (reticulocytes), while RBCs have short lives and, therefore, may become hemolysed thereby activating release of soluble receptors and signal transducing molecules, including CLNK. The latter is the third member of the SLP-76 adaptor protein family and is expressed in cytokine-activated hematopoietic cells including mast cells and NK cells. ${ }^{11,22}$ As other family members, CLNK contains 3 protein interaction domains, including amino terminus tyrosine residues, which are phosphorylated by Src family PTKs and also Syk. ${ }^{23}$ The SLP-76-related adaptor molecule CLNK physically and functionally interacts with HPK-1 in immunoreceptor-activated hemopoietic cells. CLNK has the capacity to substitute at least partially for SLP-76 during immunoreceptor signaling, even though it binds to a distinct set of partners. $^{14}$

The pathophysiological mechanisms of ineffective erythropoiesis in $\beta$-TM could be the result of several mechanisms of which the final consequence is the arrest of maturation and increased apoptosis of erythroblasts during their terminal differentiation stage. ${ }^{25}$ CLNK can function as a signaling scaffold downstream of multiple immune receptors. ${ }^{14,22,23}$ Other findings on the transcriptional regulation of the CLNK gene helps to understand how lineage-specific immunoreceptor signaling pathways are established and regulated during activation and differentiation of various hematopoietic cells. ${ }^{24}$ Increased level of several inflammatory cytokines has been reported in $\beta$-TM and may contribute to ineffective erythropoiesis, through anemia associated with chronic disease. ${ }^{25}$

Our results also showed the typical picture of iron overload state and anemia in $\beta$-TM, including higher serum iron, TS\%, ferritin, and EIBC and lower $\mathrm{Hb}$ and PCV in $\beta$-TM as compared with controls. Our findings are in accordance with Kuldeep et al., $(2011)^{26}$ who found 
a significant increase in serum iron in patients with $\beta$-TM as compared with healthy controls. These results are also in accordance with those of previous studies. ${ }^{3,27}$ The predominant mechanism driving the iron loading process includes transfusion therapy and enhanced intestinal absorption secondary to ineffective erythropoiesis. ${ }^{2}$ Serum ferritin measurement and noninvasive imaging techniques are available to diagnose iron overload, quantify its extent in different organs, and monitor clinical response to therapy. ${ }^{2}$ Higher levels of serum ferritin (especially over $2500 \mu \mathrm{g} / \mathrm{l})$ are associated with iron-related organ failure particularly in the liver and heart. ${ }^{27}$ This organ damage is not only related to oxidative stress end products, ${ }^{18}$ but also to the direct effect of iron overload on the tissues. In the current study, serum CLNK was significantly associated with number of transfusions, ferritin and iron. The overall positive correlation between CLNK and iron status parameters such as ferritin indicates the dependence of the CLNK level on iron overload status. In patients with transfusion-dependent $\beta$-TM, red blood cell transfusion is the mainstream management although regular blood transfusions may cause iron overload leading to organ damage. ${ }^{28}$ A positive association between duration of blood transfusion and degree of body iron was observed previously. ${ }^{29}$ Furthermore, some studies reported positive correlations between serum ferritin and duration of transfusion and age of the patients. ${ }^{29}$

Moreover, the interaction between CLNK and iron overload biomarkers may be explained by the formation of microvesicles in RBCs. Bailey et al. (2007) ${ }^{7}$ found that the cyclic adenosine monophosphate (cAMP)-dependent pathway plays a role in $\gamma$-globin gene (HBG) expression in adult erythroblasts in $\beta$-thalassaemia. Their results also demonstrate that the cAMP-dependent pathway is augmented by multiple cytokines, which play a role in regulating $\mathrm{Hb}$ expression in $\beta$-TM. ${ }^{7}$ Hemoglobinopathies such as sickle cell disease and thalassemia are accompanied by a substantial increase in microvesicle levels. ${ }^{16,30}$ Phosphorylation of band 3 is 
associated with clustering and correlates with microvesicle formation during storage and in the RBCs of patients with thalassemia intermedia. ${ }^{16,31}$ The involvement of various signaling pathways in RBC vesiculation is supported by the relative large numbers of signaling proteins in microvesicles obtained from the plasma of a healthy donors. ${ }^{32}$ RBCs generate microvesicles to remove damaged cell constituents such as oxidized $\mathrm{Hb}$ and damaged membrane constituents, thereby prolonging the lifespan of RBCs. Damage to $\mathrm{Hb}$, in combination with altered phosphorylation of membrane proteins such as band 3, lead to a weakening of the binding between the lipid bilayer and the cytoskeleton, thereby increasing membrane budding and microparticle shedding. The contribution of RBC-derived microvesicles to inflammation, thrombosis and autoimmune reactions emphasizes the need for a better understanding of the mechanisms and consequences of microvesicle generation. ${ }^{33}$ These factors may underpin the associations among CLNK and RBCs, which undergo hemolysis in $\beta$-TM patients.

Limitations of the study

The limitation of the study was the smaller sample size. Therefore, it is considered a pilot study and further studies on a larger cohorts are warranted.

\section{Conclusion}

In the present study we introduced a new biomarker (CLNK) to diagnose $\beta$-TM and iron overload in $\beta$-TM. The serum CLNK concentrations are higher in thalassemic patients as compared with controls. CLNK level depends on number of transfusion, Hb, ferritin, and iron.

Competing interests: No conflict of interest. 
Funding: No specific funding for this research. Self-funded.

Ethical approval: The ethics committee of Kufa University approved this study (REC number: 1321).

Acknowledgements: None.

References

1. Cao A, Galanello R, Origa R. Beta-Thalassemia. Orphanet J Rare Dis 2013;5:11.

2. Taher A, Saliba AN. Iron overload in thalassemia: different organs at different rates. Hematology Am Soc Hematol Educ Program 2017;2017(1): 265-71.

3. Abdulzahra MS, Al-Hakeim HK, Ridha MM. Study of the effect of iron overload on the function of endocrine glands in male thalassemia patients. Asian J Transfus Sci 2011;5(2):127-31.

4. Hagag AA, Elfrargy MS, Gazar RA, El-Lateef AEA. Therapeutic value of combined therapy with deferasirox and silymarin on iron overload in children with beta thalassemia. Mediterr J Hematol Infect Dis 2013;5:1-7.

5. Jemaà M, Fezai M, Bissinger R, Lang F. Methods employed in cytofluorometric assessment of eryptosis, the suicidal erythrocyte death. Cell Physiol Biochem 2017;43(2):431-444.

6. Sankaran VG, Orkin SH. The switch from fetal to adult hemoglobin. Cold Spring Harb Perspect Med 2013;3(1):a011643. 
7. Bailey L, Kuroyanagi Y, Franco-Penteado CF, Conran N, Costa FF, Ausenda S, Cappellini MD, Ikuta T. Expression of the gamma-globin gene is sustained by the cAMP-dependent pathway in beta-thalassaemia. Br J Haematol 2007;138(3):382-95.

8. Sands WA, Palmer TM. Regulating gene transcription in response to cyclic AMP elevation. Cell Signal 2008;20(3):460-6.

9. De Franceschi L, Fumagalli L, Olivieri O, Corrocher R, Lowell C A, Berton G. Deficiency of Src family kinases Fgr and Hck results in activation of erythrocyte $\mathrm{K} / \mathrm{Cl}$ cotransport. J Clin Invest 1997; 99(2): 220-7.

10. Parsons SJ, Parsons JT. Src family kinases, key regulators of signal transduction. Oncogene 2004;23(48):7906-9.

11. Cao MY, Davidson D, Yu J, Latour S, Veillette A. Clnk, a novel SLP-76-related adaptor molecule expressed in cytokine stimulated hemopoietic cells. J Exp Med 1999 (190):152734.

12. Majka M, Janowska-Wieczorek A, Ratajczak J, et al. Numerous growth factors, cytokines, and chemokines are secreted by human CD34(+) cells, myeloblasts, erythroblasts, and megakaryoblasts and regulate normal hematopoiesis in an autocrine/paracrine manner. Blood 2001;97(10):3075-85.

13. Boomer JS, Tan TH. Functional interactions of HPK1 with adaptor proteins. J Cell Biochem 2005;95:34-44.

14. Yu J, Riou C, Davidson D, et al. A: Synergistic regulation of immunoreceptor signaling by SLP-76-related adaptor Clnk and serine/threonine protein kinase HPK-1. Mol Cell Biol 2001;21:6102-12. 
15. Ferru E, Giger K, Pantaleo A, et al. Regulation of membrane-cytoskeletal interactions by tyrosine phosphorylation of erythrocyte band 3. Blood 2011;117(22):5998-06.

16. Ferru E, Pantaleo A, Carta F, et al. Thalassemic erythrocytes release microparticles loaded with hemichromes by redox activation of p72Syk kinase. Haematologica 2014;99:570-8.

17. Cluitmans JC, Gevi F, Siciliano A, et al. Red blood cell homeostasis: pharmacological interventions to explore biochemical, morphological and mechanical properties. Front Mol Biosci 2016;3:10.

18. Al-Hakeim HK, Auda FM, Ali BM. Lack of correlation between non-labile iron parameters, total carbonyl and malondialdehyde in major thalassemia. $J$ Clin Biochem Nutr 2014;55(3):203-6.

19. Al-Hakeim HK, Alhillawi ZH. Effect of serum fibroblast growth factor receptor 2 and CAPS proteins on calcium status in $\beta$-thalassaemia major patients who are free from overt inflammation. Growth Factors 2018;36:178-85.

20. Freeman V, Arneson W, Hemoglobin Production, Disorders, and Testing. p:185. In: Anderson W. and Brickell J. "Clinical Chemistry: A Laboratory Prospective" (2007) Daves Co., USA.

21. Bewick V, Cheek L, Ball J. Statistics review 13: receiver operating characteristic curves. Critical Care 2004;8(6):508-12.

22. Goitsuka R, Kanazashi H, Sasanuma H, et al.A BASH/SLP-76-related adaptor protein MIST/Clnk involved in $\operatorname{IgE}$ receptor-mediated mast cell degranulation. Int Immunol 2000;12:573-80. 
23. Goitsuka R, TatsunoA, Ishiai M, Kurosaki T, Kitamura D. MIST functions through distinct domains in immunoreceptor signaling in the presence and absence of LAT. J Biol Chem 2001;276:36043-50.

24. Sasanuma H, Tatsuno A, Tsuji K, et al. Transcriptional regulation of SLP-76 family hematopoietic cell adaptor MIST/Clnk by STAT5.Biochem Biophys Res Commun 2004;321:145-53.

25. Ribeil J-A, Arlet J-B, Dussiot M, Moura IC, Courtois G, Hermine O: Ineffective erythropoiesis in $\beta$-thalassemia. Sci World J 2013;2013:394295.

26. Kuldeep KG, Amit M, Archana T. Production ofreactive oxygen species, It's effects, drugs andplant extract used as an antioxidant, chelatoron thalassemic patients: A Review. Int J of Pharma Sci Res 2011; 2(9):2278-85.

27. Suriapperuma T, Peiris R, Mettananda C, Premawardhena A, Mettananda S. Body iron status of children and adolescents with transfusion dependent $\beta$-thalassaemia: trends of serum ferritin and associations of optimal body iron control. BMC Res Notes 2018;11(1):547.

28. Prati D. Benefits and complications of regular blood transfusion in patients with betathalassaemia major. Vox Sang 2000;79:129-37.

29. Karunaratna AMDS, Ranasingha JGS, Mudiyanse RM. Iron overload in beta thalassemia major patients. Int J Blood Transfus Immunohematol 2017;7:33-40.

30. Camus SM, De Moraes JA, Bonnin P, et al. Circulating cell membrane microparticles transfer heme to endothelial cells and trigger vasoocclusions in sickle cell disease. Blood $2015 ; 125: 3805-14$.

31. Azouzi, S., Romana, M., Arashiki, N., et al. Band 3 phosphorylation induces irreversible alterations of stored red blood cells. Am J Hematol 2018;93:E110-E112. 
32. Bosman GJ, Lasonder E, Groenen-Döpp YA, Willekens FL, Werre JM. The proteome of erythrocyte-derived microparticles from plasma: new clues for erythrocyte aging and vesiculation. J Proteomics 2012;76:203-10.

33. Leal JKF, Adjobo-Hermans MJW, Bosman GJCGM. Red blood cell homeostasis: mechanisms and effects of microvesicle generation in health and disease. Front Physiol 2018;9:703. 
Table 1. Socio-demographic, clinical and biomarker data in TM patients as compared with healthy controls.

\begin{tabular}{|c|c|c|c|c|c|}
\hline Parameter & Control & Patients & F or $\chi^{2}$ & df & p-value \\
\hline $\begin{array}{ll}\operatorname{Sex} & (\mathrm{M} / \mathrm{F})\end{array}$ & $15 / 15$ & $27 / 33$ & 0.20 & 1 & 0.654 \\
\hline Age & $8.8 \pm 2.6$ & $7.2 \pm 3.0$ & 6.76 & $1 / 88$ & 0.011 \\
\hline $\mathrm{Hb}$ & $13.47 \pm 0.99$ & $7.65 \pm 2.03$ & 219.35 & $1 / 88$ & $<0.001$ \\
\hline PCV & $41.03 \pm 2.77$ & $22.43 \pm 6.55$ & 220.82 & $1 / 88$ & $<0.001$ \\
\hline Iron $\quad \mu \mathrm{M}$ & $28.6 \pm 12.8$ & $39.8 \pm 13.2$ & 14.54 & $1 / 88$ & $<0.001$ \\
\hline TIBC $\quad \mu \mathrm{M}$ & $63.87 \pm 10.39$ & $71.22 \pm 19.00$ & 3.89 & $1 / 88$ & 0.052 \\
\hline UIBS $\quad \mu \mathrm{M}$ & $35.27 \pm 11.65$ & $31.46 \pm 17.45$ & 1.17 & $1 / 88$ & 0.283 \\
\hline TS\% & $44.49 \pm 17.28$ & $57.31 \pm 17.231$ & 11.03 & $1 / 88$ & 0.001 \\
\hline Transferrin mg/L & $160.47 \pm 26.12$ & $178.94 \pm 47.73$ & 3.89 & $1 / 88$ & 0.052 \\
\hline Ferritin $* \mathrm{mg} / \mathrm{L}$ & $59.0-227.0$ & $51.8-9598.0$ & 256.61 & $1 / 88$ & $<0.001$ \\
\hline EIBS* $\quad \mathrm{mmol}$ & $8.44-32.46$ & $7.41-1372.51$ & 256.61 & $1 / 88$ & $<0.001$ \\
\hline CLNK* $\quad \mathrm{ng} / \mathrm{ml}$ & $1.95 \pm 3.06$ & $6.24 \pm 4.78$ & 20.89 & $1 / 88$ & $<0.001$ \\
\hline zCLNK+zFerritin (z scores)* & $-1.07 \pm 0.48$ & $0.54 \pm 0.72$ & 122.92 & $1 / 88$ & $<0.001$ \\
\hline zCLNK+zFerritin+zIron (z scores)* & $-1.06 \pm 0.52$ & $0.53 \pm 0.71$ & 120.71 & $1 / 88$ & $<0.001$ \\
\hline
\end{tabular}

Results expressed as mean \pm standard deviation or as minimum and maximum

(*): Processed in Ln transformation when compared.

zCLNK+zFerritin: computed as z transformation of Ln transformation of CLNK (zCLNK) plus zLnFerritin. zLnCLNK+zLnFerritin+zLnIron: computed as zLnCLNK plus zLnFerritin plus zLnIron. 
Table 2. Results of multivariate GLM analysis with the biomarkers as dependent variables and diagnosis as explanatory variable while adjusting for extraneous variables.

\begin{tabular}{|c|c|c|c|c|c|c|}
\hline Tests & Dependent Variables & $\begin{array}{l}\text { Explanatory } \\
\text { variables }\end{array}$ & $\mathbf{F}$ & df & p-value & Partial $\eta^{2}$ \\
\hline \multirow[t]{3}{*}{ Multivariate } & \multirow[t]{3}{*}{ CLNK and zCLNK+zFerritin+zLnIron } & Diagnosis & 73.85 & $5 / 85$ & $<0.001$ & 0.635 \\
\hline & & Sex & 0.49 & $5 / 85$ & 0.612 & 0.011 \\
\hline & & age & 2.95 & $5 / 85$ & 0.058 & 0.065 \\
\hline \multirow{2}{*}{$\begin{array}{l}\text { Tests for between-subject } \\
\text { effects }\end{array}$} & CLNK & Diagnosis & 20.65 & $1 / 86$ & $<0.001$ & 0.194 \\
\hline & zCLNK+zFerritin+zIron & Diagnosis & 128.04 & $1 / 86$ & $<0.001$ & 0.598 \\
\hline \multirow[t]{3}{*}{ Univariate } & \multirow[t]{3}{*}{ CLNK } & Model & 11.79 & $2 / 87$ & $<0.001$ & 0.213 \\
\hline & & Diagnosis & 0.99 & $1 / 87$ & 0.321 & 0.011 \\
\hline & & Ferritin & 2.38 & $1 / 87$ & 0.127 & 0.027 \\
\hline Univariate & CLNK & $\begin{array}{c}\text { Model } \\
\text { Ferritin }\end{array}$ & 22.59 & $1 / 88$ & $<0.001$ & 0.204 \\
\hline
\end{tabular}

zCLNK+zFerritin+zIron: computed as z transformation of Ln transformation of CLNK (zCLNK) plus zLnFerritin plus zLnIron. 
Table 3. Results of the ROC curve and bootstrapped AUC analysis

\begin{tabular}{|l|c|c|}
\hline \multicolumn{1}{|c|}{ Parameter } & $\begin{array}{c}\text { Bootstrapped } \\
\text { AUC (n=2000) }\end{array}$ & Bootstrap 95\% CI \\
\hline CLNK & 0.738 & $0.622-0.859$ \\
\hline Ferritin & 0.978 & $0.940-1.000$ \\
\hline zCLNK+zFerritin & 0.952 & $0.907-0.987$ \\
\hline zCLNK+zFerritin+zIron & 0.948 & $0.896-0.986$ \\
\hline
\end{tabular}

$\mathrm{zCLNK}+\mathrm{zFerritin}$ : computed as z transformation of Ln transformation of CLNK (zCLNK) plus zLnFerritin. zLnCLNK+zLnFerritin+zLnIron: computed as zLnCLNK plus zLnFerritin plus zLnIron. 